\title{
5 Erfolgswirkung und Herausforderungen digitaler Geschäftsmodellentwicklung
}

M. Böhm, S. Müller, H. Krcmar, I. Welpe

Unternehmen beschäftigen sich insbesondere aus drei Gründen bislang noch nicht mit dem Thema Digitalisierung: Mangel an Budget für Digitalisierungsinitiativen (50\%), Fehlen der dafür notwendigen Fähigkeiten (38\%), sowie mangelnde Unterstützung seitens des Managements $(30 \%)$. Darüber hinaus sehen $30 \%$ der Unternehmen für Digitalisierungsaktivitäten keine Notwendigkeit. Als sinnvolle Fördermaßnahmen werden insbesondere eine Sensibilisierung für die Potenziale der Digitalisierung, Unterstützung bei der Identifikation von Ideen sowie die Förderung von Innovationspartnerschaften gesehen. ${ }^{11}$

${ }^{11}$ Die hier vorgestellten Ergebnisse beruhen auf einer Studie (Müller, Böhm, Schröer, Bakhirev, Baiasu, Krcmar \& Welpe, 2016) im Auftrag der Expertenkommission Forschung und Innovation.

(C) Der/die Autor(en) 2018

G. Oswald und H. Krcmar (Hrsg.), Digitale Transformation, Informationsmanagement und digitale Transformation, https://doi.org/10.1007/978-3-658-22624-4_5 


\subsection{Einleitung}

Digitalisierung und Automatisierung sind zwei Seiten derselben Medaille. Entsprechend wird mit dem Digitalisierungsbegriff schnell der Wegfall von Arbeitsplätzen assoziiert. Die Diskussionen zur Frage der Notwendigkeit der Einführung von Roboter-Steuern ${ }^{12}$ und der Einführung eines Grundeinkommens für jeden Bürger ${ }^{13}$ zeigen, dass die Digitalisierung als eine zentrale gesellschaftliche Herausforderung wahrgenommen wird. Als Vorboten gesellschaftlicher Veränderungen lassen sich die Umwälzungen interpretieren, die sich durch die Digitalisierung in der Wirtschaft vollziehen. Die Übernahme der Lebensmittelkette Whole Foods durch den eCommerce-Riesen und Cloud-Service-Anbieter Amazon oder das Engagement der Google-Mutter Alphabet im Bereich des autonomen Fahrens zeigen, wie sich durch die Digitalisierung Branchengrenzen verschieben oder sogar auflösen.

Etablierte Unternehmen stehen vor der Herausforderung, sich digital zu transformieren, um damit den Wandel in ihrer Branche proaktiv zu gestalten oder sich ihm zumindest anzupassen. Burberry ist ein Beispiel für ein traditionelles Unternehmen, das mit Hilfe einer digitalen Transformation seine Wettbewerbsposition signifikant verbessern konnte. Burberry gelang es, seinen Umsatz seit 2006 nahezu zu vervierfachen und sich mit einer Umsatzrendite von 14,1 \% zu einem hochprofitablen Unternehmen der Modebranche zu entwickeln (Burberry, 2017).

Im Folgenden werden zunächst die Ergebnisse einer quantitativen Umfrage vorgestellt, anhand derer der Frage nachgegangen wird, vor welche Herausforderungen eine digitale Transformation etablierte Unternehmen stellt. Anschließend werden die Erfolgsaussichten verschiedener Geschäftsmodellmuster in einem zunehmend von digitalen Technologien geprägten Wettbewerb analysiert. Abschließend wird diskutiert, wie Unternehmen unterstützt werden können, um digitale Transformationsinitiativen erfolgreich initiieren und durchführen zu können und damit auch in Zukunft wettbewerbsfähig zu bleiben.

\subsection{Herausforderungen einer digitalen Transformation}

Für die Erhebung der Umfragedaten wurde ein Marktforschungsinstitut beauftragt. Um einen Vergleich zwischen den von Unternehmen wahrgenommenen Herausforderungen in unterschiedlichen Ländern ziehen zu können, wurde die Umfrage in Deutschland und den USA

\footnotetext{
${ }^{12}$ Siehe hierzu: www.faz.net/aktuell/wirtschaft/netzwirtschaft/automatisierung-bill-gates-fordert-roboter-steuer14885514.html, zugegriffen am 18.07.2017

${ }^{13}$ Siehe hierzu: www.sueddeutsche.de/wirtschaft/sz-wirtschaftsgipfel-siemens-chef-plaediert-fuer-ein-grundeinkommen-1.3257958, zugegriffen am 18.07.2017
} 
durchgeführt. Insgesamt haben pro Land jeweils 190 Unternehmen an der Befragung teilgenommen, die den Fragebogen vollständig ausgefüllt haben.

Der Befragung vorausgegangen ist ein Pretest unter 29 Umfrageteilnehmern. Die Ergebnisse des Pretests haben zu leichten Anpassungen des Fragebogens geführt. Die Zielgruppe waren Führungskräfte, die an unternehmensstrategischen Entscheidungen beteiligt sind, bzw. Einblick darauf haben. Im Idealfall handelte es sich dabei um Geschäftsführer, Vorstände, Aufsichtsräte oder Personen auf C-Level. Zulässig waren auch Bereichs- und Abteilungsleiter sowie Führungskräfte mit IT-Verantwortung. Die Umfrage wurde zwischen dem 14. und 22. Januar 2016 durchgeführt. Es wurden 2.833 Teilnehmer in den USA und 1.536 Teilnehmer in Deutschland eingeladen. Ein wesentliches Kriterium war, dass sich die Teilnehmer bereits mit dem Thema Digitalisierung beschäftigt haben um den Fragebogen sinnvoll ausfüllen zu können. Hierfür wurde dem Fragebogen eine Selektionsfrage hinzugefügt, durch welche 460 (US) und 162 (DE) Teilnehmer aussortiert wurden. Von den eingeladenen Personen haben 115 die Umfrage vorzeitig abgebrochen.

Zur Untersuchung der mit dem Thema digitale Transformation assoziierten Herausforderungen wurden aus den teilnehmenden Unternehmen jene ausgewählt, die noch keine digitale Transformationsinitiative gestartet hatten. Insgesamt haben 37 der teilnehmenden deutschen und 27 der teilnehmenden US-amerikanischen Unternehmen noch keine digitale Transformationsinitiative gestartet. Diese Unternehmen wurden gezielt nach den Gründen für ihre bisherige Zurückhaltung gefragt.

Abbildung 4 zeigt, dass sich ein großer Teil (35\%) der deutschen Unternehmen zumindest in grundlegender Art und Weise bereits mit dem Thema Digitalisierung beschäftigt hat. Dies trifft ebenfalls auf $30 \%$ der US-amerikanischen Unternehmen zu. Besorgniserregend ist, dass sich, unabhängig vom untersuchten Land, ein etwa ebenso großer Teil der befragten Unternehmen noch überhaupt nicht mit den Implikationen der Digitalisierung für das eigene Geschäft auseinandergesetzt hat. Damit liegt eine der großen Hürden für die Digitalisierung in der deutschen Wirtschaft auf der Hand: Ein fehlendes Bewusstsein für das Thema Digitalisierung oder ein mangelnder Wille sich damit auseinanderzusetzen.

Gründe dafür, dass noch keine konkreten Digitalisierungsinitiativen geplant wurden, lassen sich auf der Seite der US-Unternehmen in der Stichprobe in einem unzureichenden Budget (63 \% stimmen zu), einer fehlenden Managementunterstützung (37\% stimmen zu) und fehlenden Fähigkeiten der Mitarbeiter (30 \% stimmen zu) finden. Auf der Seite der deutschen Umfrageteilnehmer lassen sich Gründe in mangelnden Fähigkeiten für die Umsetzung einer digitalen 
Transformationsinitiative (43\% stimmen zu) sowie einem mangelnden Budget (41\% stimmen zu) sehen. Etwa ein Viertel der deutschen Unternehmen und zwei Fünftel der amerikanischen Unternehmen geben an, dass aktuell keine Notwendigkeit zur Digitalisierung besteht. Dies birgt die Gefahr, dass Veränderungen im Wettbewerb erst spät erkannt werden. Eine geringe Motivation der Mitarbeiter zur Veränderung scheint hingegen kein Grund für das Ausbleiben einer Transformationsinitiative zu sein.

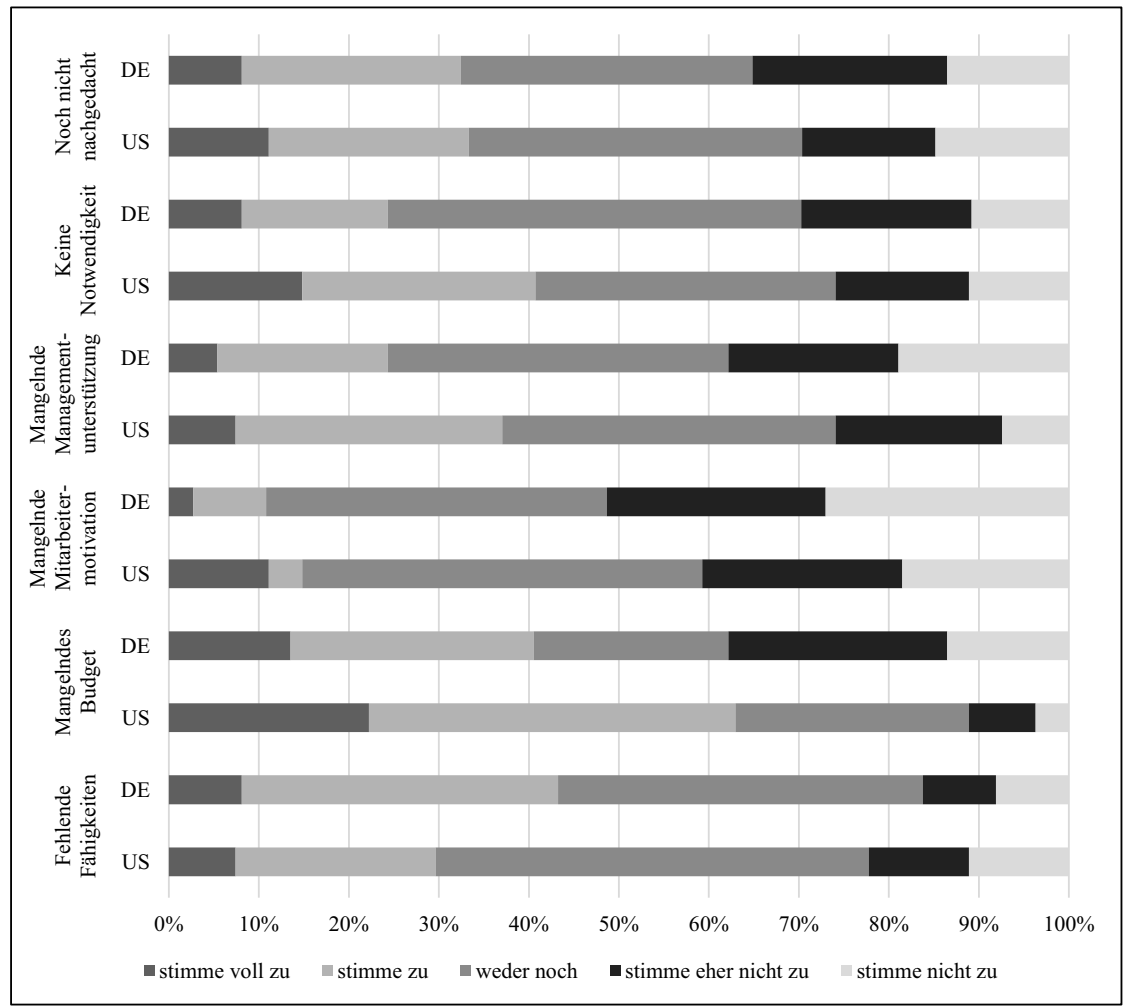

Abbildung 4: Hemmnisse für Digitalisierungsaktivitäten (Quelle: Eigene Darstellung $\left(\mathrm{n}_{\mathrm{DE}}=37, \mathrm{n}_{\mathrm{US}}=27\right)$ )

Dass sich fehlendes oder unzureichende Budget als eine der zentralen Herausforderungen erweist, denen sich die Unternehmen in der Stichprobe stellen müssen, ist nicht verwunderlich. Investitionen in digitale Technologien sind anders zu behandeln als traditionelle Investitionen, beispielsweise in Maschinen. Investitionen in digitale Technologien haben eher den Charakter von Forschungs- und Entwicklungsprojekten. Darüber hinaus werden Digitalisierungsinitiati- 
ven in Großunternehmen auch als IT-getriebene Projekte des organisationalen Wandels verstanden. Dies spiegelt sich auch in der Umfrage wider. Bezogen auf die gesamte Stichprobe fassen $63 \%$ der befragten Unternehmen Investitionen in digitale Technologien als transformativ im Sinne einer weitreichenden Veränderung des Unternehmens auf. Derartige Investitionen haben größere Auswirkungen auf Geschäftsprozesse und Mitarbeiter. Gut 60 \% der Unternehmen finden Investitionen in digitale Technologien schwieriger zu bewerten als traditionelle Investitionen. Eine Wirtschaftlichkeitsbetrachtung derartiger Investitionen darf zwar nicht außer Acht gelassen werden, jedoch hat der Großteil der befragten Unternehmen Probleme, diese Investitionen objektiv zu bewerten. Dies erschwert es, eine Investition in digitale Technologien gegenüber Vorgesetzten und Mitarbeitern zu rechtfertigen. $45 \%$ der Unternehmen sehen hier eine zentrale Herausforderung.

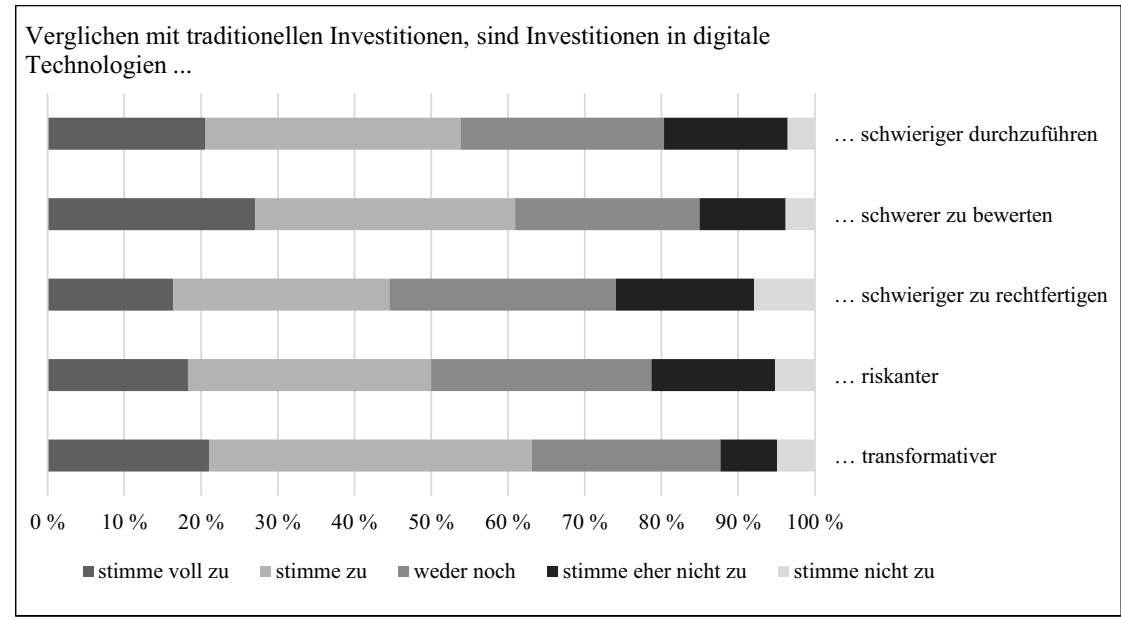

Abbildung 5: Investitionen in digitale Technologien (Quelle: Eigene Darstellung $(\mathrm{n}=366)$ )

Etwa zwei Drittel der Unternehmen bewerten Investitionen in digitale Technologien dagegen als schwieriger. Dies könnte auf mangelnde Erfahrung mit derartigen Projekten sowie fehlende Mitarbeiter mit entsprechender Technologiekompetenz zurückgeführt werden. Auf Grund der Schwierigkeit hinsichtlich der Durchführung und Bewertung empfindet die Hälfte der Unternehmen Digitalisierungsaktivitäten als eine im Vergleich zu anderen Optionen riskantere Investition.

Wie eine Umfrage der GfK zeigt, spiegelt sich diese Auffassung in den tatsächlichen Investitionen der Unternehmen wider. Die Umfrage unter kleinen und mittelständischen Unternehmen hat ergeben, dass lediglich vereinzelte Unternehmen signifikante (mehr als $50 \%$ des 
gesamten Investitionsbudgets) Investitionen in digitale Technologien tätigen (vgl. Abbildung 6). Der Großteil investiert unter $10 \%$. Hier ergeben sich auch in den kommenden drei Jahren nur geringfügige Veränderungen (GfK, 2014, S. 31f.).

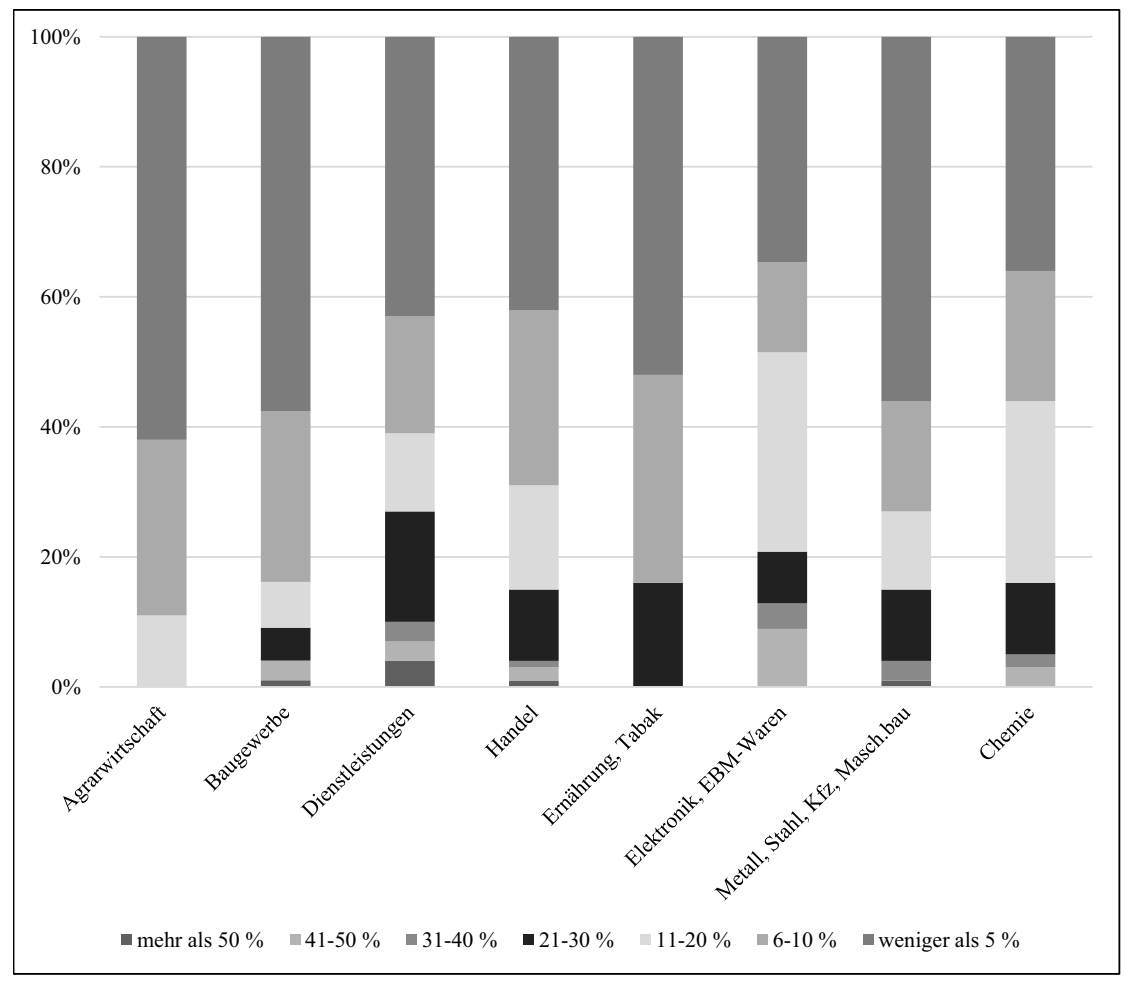

Abbildung 6: Geschätzter Investitionsbedarf für digitale Technologien in KMUs (Quelle: Eigene Darstellung in Anlehnung an GfK (2014)), S. 31)

\subsection{Implikationen digitaler Geschäftsmodelle}

Eine Betrachtung der mit Initiierung und Durchführung digitaler Transformationsinitiativen in Zusammenhang stehenden Herausforderungen alleine hilft nicht bei der Beantwortung der Frage, ob eine entsprechende Investition von Zeit und finanziellen Mitteln lohnenswert ist. Daher stellt die Umfrage den mit der Transformation in Verbindung gebrachten Herausforderungen die damit realisierten Vorteile gegenüber. 


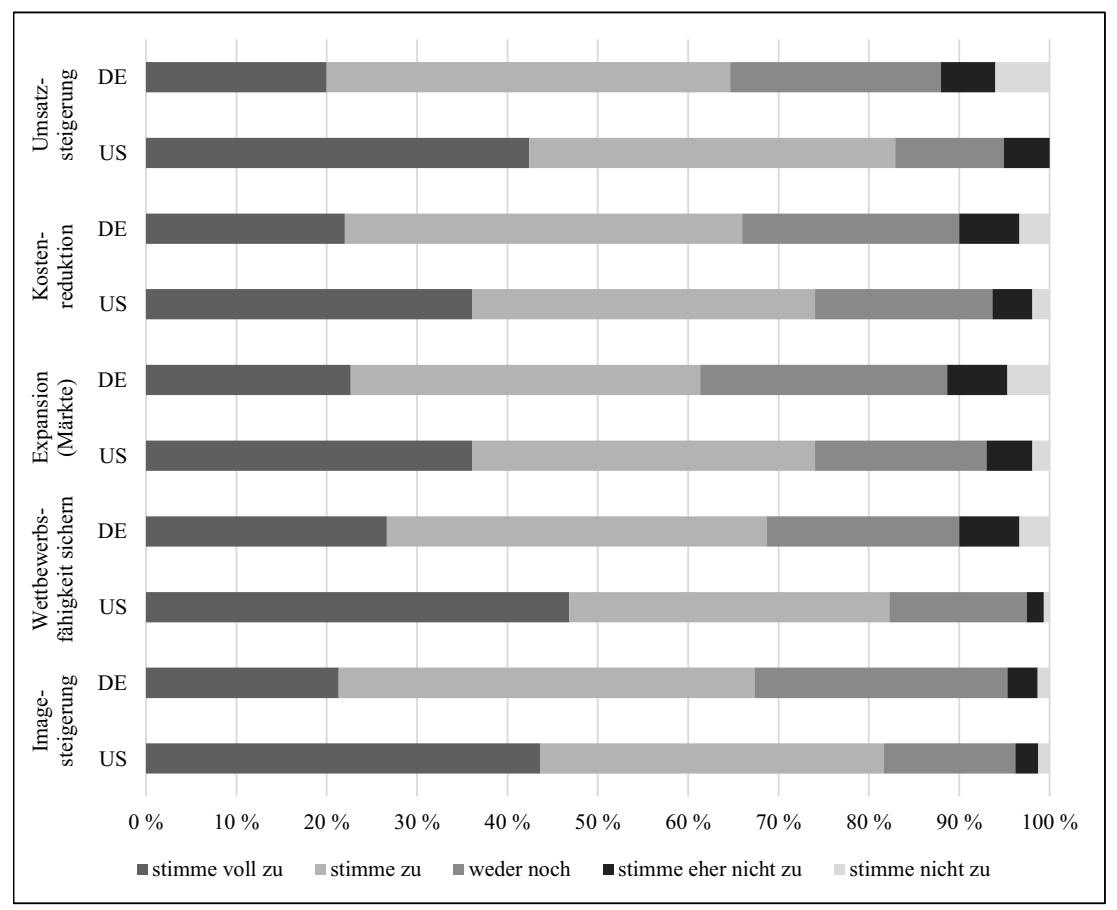

Abbildung 7: Implikationen der Digitalisierungsaktivitäten (Quelle: Eigene Darstellung $\left(\mathrm{n}_{\mathrm{DE}}=150, \mathrm{n}_{\mathrm{US}}=158\right)$ )

Rund drei Viertel der befragten Unternehmen schätzen, dass es ihnen durch ihre Digitalisierungsaktivitäten gelungen ist (75\%) bzw. gelingen wird (77\%), ihre Wettbewerbsfähigkeit für die kommenden fünf bis zehn Jahre zu sichern. Wie Abbildung 7 zeigt, bewerten US-amerikanische Unternehmen die mit ihrer Digitalisierung in Zusammenhang stehenden Vorteile positiver, als die deutschen Unternehmen in der Stichprobe. So geben mehr amerikanische Firmen an, ihre Erfolgsziele zu erreichen. Mehr als zwei Drittel der Unternehmen berichten, dass Sie durch Digitalisierungsmaßnahmen den Umsatz erheblich steigern oder die Kosten signifikant senken konnten. Darüber hinaus gelang es der Mehrheit dieser Unternehmen, neue Märkte zu erschließen sowie das Unternehmensimage zu verbessern. Über alle Erfolgsdimensionen hinweg ist der Anteil der Unternehmen, die keinen positiven Zusammenhang zwischen dem Erfolg des Unternehmens und dessen Digitalisierung sehen sehr klein und liegt, abgesehen von den Erfolgsdimensionen „Umsatzsteigerung“ und „Expansion in neue Märkte“, bei $10 \%$ und weniger. 


\subsection{Geschäftsmodelle erfolgreicher Startups}

Mit einer digitalen Transformation wird häufig eine substantielle Veränderung eines bestehenden oder die Etablierung eines neuen Geschäftsmodells verbunden. Vor dem Hintergrund der im vorhergehenden Abschnitt diskutierten Geschäftspotentiale einer digitalen Transformation, stellt sich die Frage, welche Geschäftsmodelle sich eignen, um in Märkten zu bestehen, die zunehmend durch digitalisierte Produkte und Dienstleistungen gekennzeichnet sind.

Auf Basis einer Analyse der Unternehmensprofile von 181 deutschen und amerikanischen Startups auf Crunchbase wurden die Erfolgsaussichten einzelner Geschäftsmodellmuster untersucht. Dabei wurden die Geschäftsmodelle anhand des St. Gallener Business Model Navigators (Gassmann, Frankenberger, \& Csik, 2014) klassifiziert und mit Hilfe einer Clusteranalyse zusammengefasst. Die Bezeichnung der Cluster richtet sich nach dem Geschäftsmodellmuster mit der größten Ladung, wobei Ladungen unter 0,4 nicht berücksichtigt wurden. Bei der Interpretation der Daten ist von einer positiven Verzerrung der Stichprobe auszugehen, da gescheiterte Unternehmen unterrepräsentiert sind. Tabelle 1 zeigt den relativen Anteil der erfolgreichen Startups je Cluster. Als Erfolgsmaße wurden dabei das Überleben des Startups sowie das jährliche Umsatzwachstum in Relation zur Unternehmensgröße (Maltz \& Saljoughian, 2013) herangezogen.

Tabelle 1: Geschäftsmodelle erfolgreicher Startups (Quelle: Eigene Darstellung)

\begin{tabular}{|l|l|l|l|l|}
\hline Cluster & n & $\begin{array}{l}\text { Schnelles } \\
\text { Wachstum }\end{array}$ & $\begin{array}{l}\text { Langsames } \\
\text { Wachstum }\end{array}$ & Überleben \\
\hline Freemium Platforms & 6 & $50 \%$ & $50 \%$ & $100 \%$ \\
\hline Experience Crowdusers & 11 & $18 \%$ & $27 \%$ & $64 \%$ \\
\hline Long Tail Subscribers & 16 & $75 \%$ & $75 \%$ & $94 \%$ \\
\hline Affiliate Markets & 23 & $52 \%$ & $65 \%$ & $78 \%$ \\
\hline Mass Customizing Orchestrators & 12 & $50 \%$ & $67 \%$ & $83 \%$ \\
\hline Innovative Platforms & 6 & $67 \%$ & $67 \%$ & $100 \%$ \\
\hline E-Commercers & 14 & $43 \%$ & $57 \%$ & $57 \%$ \\
\hline E-Commerce Affiliates & 29 & $45 \%$ & $45 \%$ & $76 \%$ \\
\hline Add-on Layers & 20 & $45 \%$ & $60 \%$ & $90 \%$ \\
\hline Crowdsourcing Platforms & 11 & $73 \%$ & $91 \%$ & $91 \%$ \\
\hline Customized Layers & 13 & $54 \%$ & $62 \%$ & $69 \%$ \\
\hline Hidden Revenue Markets & 20 & $25 \%$ & $25 \%$ & $75 \%$ \\
\hline
\end{tabular}


Das Cluster Freemium Platform umfasst Unternehmen, die im Kern das Freemium- bzw. die einer Plattform zuzuordnenden Geschäftsmodellmuster Orchestrator, Two-Sided Market und Long Tail verfolgen. Erstere bieten einen kostenlosen Basisdienst an, um eine breite Kundenbasis zu gewinnen. Erlöse werden durch kostenpflichtige Zusatzangebote generiert. Orchestrator koordinieren Wertschöpfungsaktivitäten verschiedener Unternehmen, um ihren Kunden ein aggregiertes Produkt anzubieten. Im Rahmen des Two-Sided-Market-Geschäftsmodells werden verschiedene Kundengruppen durch eine Plattform bedient. Dabei ist die Plattform für eine der Kundengruppen nur interessant, wenn auch die andere in ausreichender Anzahl vertreten ist. Aus diesem Grund werden einer Kundengruppe meist kostenlose Dienste angeboten. Ein typisches Beispiel für dieses Geschäftsmodell ist die Suchmaschine Google. Die Grundlage beider Geschäftsmodellmuster ist eine Vermittlungsplattform. Das Long-Tail-Muster wird benutzt, wenn der Gewinn über viele kleine Zahlungen mit einer geringen Marge erzielt wird. Insofern umfasst dieses Cluster Unternehmen, die Leistungen verschiedener Anbieter aggregieren und auf ihrer Plattform als kostenfreien Basisdienst und mit kostenpflichtigen Zusatzdiensten anbieten. Während alle Unternehmen in der Stichprobe überleben, weist nur die Hälfte der Unternehmen ein starkes Umsatzwachstum auf. Die Anzahl der Unternehmen in diesem Cluster ist mit sechs aber auch niedrig. Es ist somit davon auszugehen, dass nur wenige Kunden bereit sind, für die Zusatzleistungen zu bezahlen. Ein typischer Vertreter des Clusters Freemium Platform ist Soundcloud, eine Plattform um Künstler mit ihren Fans zu verbinden. Sie bietet einen freien Upload von 3 Stunden Audiomaterial für Künstler an. Mehr Audiomaterial oder ein aussagekräftigeres Profil sind kostenpflichtig.

Das Cluster Experience Crowduser umfasst Unternehmen, die die beiden Geschäftsmodellmuster Experience Selling und Crowdsourcing verfolgen. Zudem benutzen sie das Muster Leverage Customer Data. Im Rahmen des Experience-Selling-Geschäftsmodells steht das Erleben des Kunden bei der Benutzung eines Angebots im Mittelpunkt. Das Ziel ist es, ein einzigartiges Erlebnis rund um das Angebot aufzubauen. Beim Crowdsourcing-Muster werden zentrale Tätigkeiten der Wertschöpfung an die „Crowd“, also entweder an die breite Öffentlichkeit oder eine ausgewählte Gruppe, ausgelagert. Hierdurch lässt sich das Potential der Masse für das eigene Unternehmen nutzen. Das Leverage-Customer-Data-Muster zeichnet sich dadurch aus, dass versucht wird, aus Kundendaten einen Vorteil zu ziehen. Dies kann wie im Falle von Google darin bestehen, anderen Unternehmen maßgeschneiderte Werbung für jeden Nutzer anzubieten. Dieses Cluster hat mit $64 \%$ nicht nur die zweitschlechteste Überlebens- sondern mit $18 \%$ auch noch die schlechteste Erfolgsrate. Dies bedeutet, dass es sehr schwierig ist, mit die- 
sem Geschäftsmodell zu überleben. Selbst wenn das Startup überlebt, stehen die Wachstumsaussichten sehr schlecht. Somit eignet sich dieses Cluster eher für Nischenmärkte. Ein weiteres Beispiel des Clusters Experience Crowduser ist Researchgate, eine Website für universitäre Forschung. Die Nutzer können mit Gleichgesinnten ihre Fachgebiete diskutieren und auch ihre Veröffentlichungen gesammelt bereitstellen.

Das Cluster Long Tail Subscriber setzt auf das Long-Tail- sowie das Subscription-Muster als zentrale Bestandteile seiner Strategie. Letzteres bezeichnet eine auf gewisse Zeit vertraglich festgelegte periodische Zahlung des Nutzers an den Bereitsteller eines Angebots. Dafür kann das Angebot im vertraglich festgelegten Rahmen genutzt werden. Diese Kombination ist bei digitalen Gütern sehr erfolgreich, da die Vervielfältigung von Informationsprodukten oder das Angebot von Softwarelösungen fast keine marginalen Kosten erzeugt. Deshalb können die Produkte sehr günstig angeboten werden und somit durch viele kleinere Zahlungen über einen längeren Zeitraum größere Gewinne erzielt werden. Adobe hat zum Beispiel seine Angebote von einmaligen Verkaufspreisen auf Abonnements umgestellt. Diese Kombination wird auch für digitale Medien eingesetzt. Da 94 \% der Nutzer dieses Clusters überlebten, kann es als sehr erfolgreich angesehen werden. Hinzu kommt, dass auch $75 \%$ der Unternehmen ein hohes Wachstum aufweisen. Somit wachsen fast alle Unternehmen in diesem Cluster sehr stark. Ein weiteres Beispiel des Clusters Long Tail Subscriber ist Babbel. Babbel bietet Abonnements zum Erlernen einer Fremdsprachekursabonnements an. Durch das digitale Geschäftsmodell können die Preise pro Kunde geringgehalten, aber über die Masse genügend Geld verdient werden.

Das Cluster Affiliate Markets nutzt neben dem Affiliate-Muster die klassischen Muster einer Plattform: Two-Sided Market, Orchestrator und Long Tail. Hinzu kommt jedoch auch noch das Aikido-Muster. Im Rahmen des Affiliation-Geschäftsmodells werden Provisionen für die Vermittlung von Kunden an Dritte ausbezahlt. Preisvergleichsportale sind ein Beispiel für die Kombination des Affiliate-Modells mit einer Plattform. Aikido hingegen bedeutet, etwas anders zu machen als die Konkurrenz in der gleichen Industrie. Das Übertragen von Geschäftsmodellen, welche sich in anderen Industrien bewährt haben, auf die eigene Industrie ist ein Beispiel für Aikido. Die Überlebensrate dieses Clusters liegt im Mittelfeld aller Cluster. Zudem weist dieses Cluster eher geringe Wachstumschancen auf. Dies kann dadurch bedingt sein, dass es sich um einen bereits eher gesättigten Markt mit großem Verdrängungswettbewerb und bereits etablierten großen Unternehmen handelt. Ein typischer Vertreter des Clusters Affiliate 
Markets ist Airbnb, welches privaten Anbietern von Übernachtungsmöglichkeiten eine Plattform zur Kundengewinnung anbietet.

Die Mass Customizing Orchestrators verbinden das Mass-Customization-Muster mit dem Layer Player, dem Orchestrator und dem Two-Sided-Market-Muster zu einem Geschäftsmodell. Mass Customization bedeutet, dass Produkte zwar in Massenfertigung produziert, aber durch eine große Auswahl an verschiedenen Optionen trotzdem bis zu einem bestimmten Grad individualisierbar sind. Wenn man eine Lösung für einen bestimmten Teil der Wertschöpfungskette in verschiedenen Industrien anbietet, benutzt man das Layer-Player-Muster. Dieses Cluster kombiniert somit ein individuelles Angebot mit einer Plattform auf der die Teilangebote einzelner Partner erhältlich sind. Es weist eine Überlebensquote von 84 \% und eine Erfolgsquote von $50 \%$ auf. Dies bedeutet, dass es in diesem Cluster durchaus möglich ist, schnell zu wachsen. Ein beispielhafter Vertreter des Clusters Mass Customizing Orchestrators ist Test Birds, welche Kunden individuelles App- oder Website-Testing anbietet, welches durch die Crowd vorgenommen wird. Somit ist Test Birds der Mittelsmann zwischen dem eigentlichen Tester und dem Kunden.

Das nächste Cluster, Innovative Platforms, setzt neben den klassischen Mustern einer Plattform, wie Two-Sided Market, Orchestrator und Revenue Sharing, vor allem auf das AikidoMuster. Somit versuchen die Unternehmen in diesem Cluster neue Ideen in das Geschäftsmodell der Plattformen zu integrieren. Dieser Versuch kann auch als durchaus erfolgreich gesehen werden, da alle Unternehmen der Stichprobe bis jetzt überlebt haben und zwei Drittel auch stark wachsen. Ein repräsentatives Beispiel des Clusters Innovative Platforms ist Instacart, eine innovative Shopping-Plattform, welche Lebensmittel von großen Partnerketten an den Kunden liefert.

Das Cluster E-Commercer ist geprägt durch die Muster E-Commerce und Direct Selling. Bei E-Commerce-Mustern werden Produkte oder Dienstleistungen über das Internet angeboten. Direct Selling bedeutet, dass die Produkte direkt von den Produzenten, also ohne Zwischenhändler, an den Kunden vertrieben werden. Dieses Cluster hat die niedrigste Überlebensquote und die zweitniedrigste Erfolgsquote. Das liegt daran, dass der E-Commerce-Markt bereits sehr gesättigt ist, was es für junge Unternehmen, welche ohne innovative Geschäftsmodelle den Markt betreten, durch starken Preiskampf sehr schwer macht. Zudem wird der Markt von einigen großen Anbietern wie Amazon und Zalando für Mode in Deutschland dominiert. Überlebenschancen bestehen nur in der Nische. 
Das Cluster E-Commerce Affiliates schlägt sich etwas besser als das E-Commerce-Cluster. Es setzt neben den durch die E-Commercer eingesetzten Mustern auch auf die Affiliate- und Long-Tail-Muster. Dadurch überleben immerhin ca. drei von vier Unternehmen in diesem Cluster. Starkes Wachstum können aber auch nur 45 \% generieren. Durch Aggregation der Angebote vieler Anbieter wird für den Kunden eine große Auswahl geschaffen. Über Links zu den Webseiten der eigentlichen Shops wird Provision erzeugt. Dieses Geschäftsmodell haben vor allem Vergleichsportale wie Idealo oder Check24 bekannt gemacht.

Die Add-on Layers nutzen die Muster Add-on, Layer Player und Subscription. Beim Addon-Muster wird ein Basisangebot relativ günstig zur Verfügung gestellt. Für ein Angebot mit mehr Optionen muss ein Aufpreis bezahlt werden. Dieses Muster wird vor allem bei SaaSProdukten oft eingesetzt. GitHub ist ein Beispiel für dieses Muster. Dort wird ein öffentliches Software-Repository gratis angeboten. Möchte man zusätzlich dazu noch ein privates Repository nutzen, muss man einen Aufpreis zahlen, der davon abhängt wie viele Projekte und Benutzerkonten benötigt werden. 90 \% der Firmen, welche dieses Geschäftsmodell benutzen, haben bis jetzt überlebt, jedoch wachsen nur $45 \%$ stark. Dies kann man darauf zurückführen, dass bei vielen Diensten die Nutzer der Basisversionen überwiegen.

Aikido, Crowdsourcing, Customer Loyalty und die bereits erwähnten Plattformmuster werden vom Cluster Crowdsourcing Platforms eingesetzt. Customer Loyalty bedeutet, dass versucht wird, durch Anreize wie Bonusprogramme oder Belohnungen für wiederholte Nutzung die Aktivität der Kunden zu steigern bzw. zu erhalten. Besonders für Crowdsourcing ist es enorm wichtig, eine aktive Community zu entwickeln. Deshalb ist die Kombination dieser zwei Muster durchaus sinnvoll. Ebenso wird für Crowdsourcing eine Plattform zum Austausch benötigt. Der Erfolg gibt den Nutzern dieses Geschäftsmodells auch Recht: Neun von zehn Firmen überleben und fast drei von vier wachsen sehr stark. Dies lässt sich eventuell dadurch begründen, dass es in den letzten Jahren einen starken Wandel vom reinen Konsumenten hin zum Dialog mit den Unternehmen gegeben hat. Aus diesem Grund sind viele Kunden bereit, Zeit und Energie in Crowdsourcing-Kampagnen zu investieren. Ein typischer Vertreter des Clusters Crowdsourcing Platforms ist DaWanda. Auf DaWanda können Nutzer ihre selbst hergestellten Produkte anbieten. Das Unternehmen erhält dafür eine Verkaufsprovision.

Die Customized Layers benutzen neben dem Layer Player auch das Subscription- und das Mass-Customization-Muster. Die Kombination aus Layer Player und Mass Customization ist vor allem in der SaaS-Industrie sehr gebräuchlich. Hier wird eine Basis-Software, welche in vielen Industrien einsetzbar ist, individuell auf die Unternehmen zugeschnitten. SAP bietet zum 
Beispiel Standard-Software an, welche sich anpassen lässt, um die Geschäftsprozesse des Kunden abzubilden. Etwas weniger als $75 \%$ der Firmen in diesem Cluster überleben. Zusätzlich weisen nur $54 \%$ starkes Wachstum auf. Ein typischer Vertreter des Clusters Customized Layers ist AdJust, welches App-Analytics für zahlreiche Industrien anbietet. Das Angebot wird auf die Bedürfnisse der Kunden zugeschnitten.

Die Hidden Revenue Markets setzen auf das Hidden-Revenue- und das Two-Sided-MarketMuster. Zudem spielen auch Affiliation und Long Tail eine Rolle. Beim Hidden-Revenue-Muster ist meist ein Angebot für eine Seite eines Two-Sided Markets kostenlos während die andere Kundengruppe bezahlen muss. Ein Beispiel hierfür ist die Google-Suche. Werbetreibende müssen dafür bezahlen, Benutzern der Suchfunktion ihre Produkte anzubieten. Die Suchmaschine an sich ist aber für die normalen Nutzer umsonst. Die Kombination aus Long Tail und Hidden Revenue lässt auf ein hohes Transaktionsvolumen schließen. Sonst wäre es nicht möglich mit geringen Zahlungen das Angebot aufrecht zu erhalten. Das Cluster schneidet mit diesem Geschäftsmodell nicht sehr gut ab. Zwar überleben drei von vier Firmen aber nur eine von vier wächst stark. Ein weiteres Beispiel für das Cluster Hidden Revenue Markets ist qLearning, eine Plattform für Studenten um sich auf Prüfungen vorzubereiten. Diese ist für die Nutzer kostenlos und wird durch Werbung finanziert.

\subsection{Unterstützung für etablierte Unternehmen}

Aus Sicht der Unternehmen, die bislang noch keine Digitalisierungsaktivitäten durchführen oder planen, sind die Fördermöglichkeiten vielfältig. Diesen Unternehmen fehlen insbesondere Informationen zu Potenzialen der Digitalisierung sowie Ansätze und Ideen. Fördermaßnahmen könnten daher auf eine initiale Beratung abzielen. Diese könnte beispielsweise durch Forschungseinrichtungen oder Kompetenzzentren angeboten werden. Forschungsnahe Kompetenzzentren könnten weiterhin dem Wunsch nach Innovationspartnerschaften nachkommen, da sich neben einer initialen Beratung auch gemeinsame Projekte anstoßen ließen. Zudem können derartige Kompetenzzentren als Plattform dienen, um Kontakte zwischen Interessenten (KMUs, Startups, etc.) zu vermitteln, aus denen Innovationspartnerschaften erwachsen können.

Aus Sicht der Unternehmen, die bislang noch keine Digitalisierungsaktivitäten durchführen oder planen, sind die Fördermöglichkeiten vielfältig. Wie Abbildung 8 zeigt, fehlen diesen Unternehmen insbesondere Informationen zu Potenzialen der Digitalisierung (gesamt 54 \%) sowie 
Ansätze und Ideen (gesamt 61 \%). Diese Funktion übernehmen bei den Unternehmen mit Digitalisierungsinitiativen häufig Beratungsunternehmen. Fördermaßnahmen könnten daher eine derartige Beratungsfunktion adressieren.

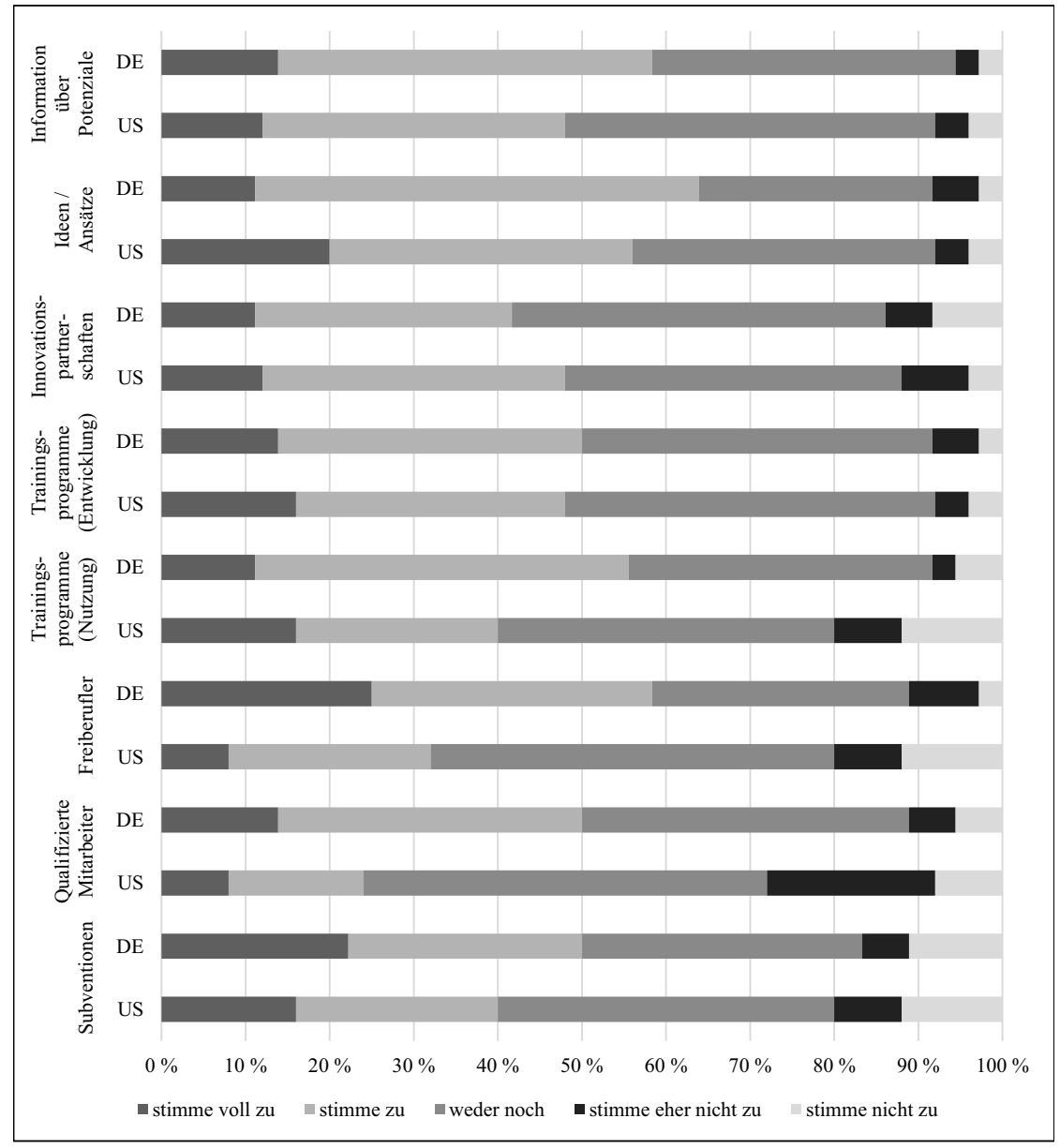

Abbildung 8: Maßnahmen zur Förderung von Digitalisierungsaktivitäten (Quelle: Eigene Darstellung $\left(\mathrm{n}_{\mathrm{DE}}=\right.$ $\left.36, \mathrm{n}_{\mathrm{US}}=25\right)$ )

Knapp die Hälfte der Umfrageteilnehmer führen auch Subventionen wie beispielsweise Steuererleichterungen als geeignete Maßnahme an. Insbesondere große Unternehmen verfügen über das notwendige Budget, um Digitalisierungsinitiativen durchzuführen. Insofern sollten 
Subventionen für die Förderungen von KMUs bzw. Startups in Betracht gezogen werden. Subventionen könnten auch in Form von Beratungsgutscheinen angeboten werden, um den Bedarf nach Informationen über Digitalisierungspotenziale und -ideen zu adressieren.

In Deutschland wird eine bessere Verfügbarkeit von qualifizierten Mitarbeitern (50\%) oder der Zugang zu kurzfristigen Personalkapazitäten beispielsweise in Form von Freiberuflern für die Realisierung der Digitalisierungsmaßnahmen (58 \%) als adäquate Fördermaßnahme angesehen. Ähnlich verhält es sich mit Trainingsprogrammen für Mitarbeiter. Die Hälfte der deutschen und $48 \%$ der US-amerikanischen Unternehmen sehen einen zusätzlichen Qualifikationsbedarf um Mitarbeiter in die Lage zu versetzen Digitalisierungsinitiativen zu entwickeln und zu implementieren. Auch für die darauffolgende Nutzungsphase bedarf es einer weiteren Qualifikation. So erachten $40 \%$ der US-amerikanischen und $56 \%$ der deutschen Unternehmen diese als notwendig.

\subsection{Literaturverzeichnis}

Burberry. (2017). Burberry Annual Report 2016/2017, London, England.

Gassmann, O., Frankenberger, K., \& Csik, M. (2014). The Business Model Navigator.

Harlow, UK: Pearson Education.

GfK. (2014). Umfrage in mittelständischen Unternehmen zum Thema Digitalisierung -

Bedeutung für den Mittelstand im Auftrag der DZ Bank.

Maltz, J., \& Saljoughian, P. (2013). How Fast Should You Be Growing? URL:

http://techcrunch.com/2013/08/24/how-fast-should-you-be-growing

Müller, S., Böhm, M., Schröer, M., Bakhirev, A., Baiasu, B., Krcmar, H., \& Welpe, I. (2016).

Geschäftsmodelle in der digitalen Wirtschaft, Studien zum deutschen

Innovationssystem, Berlin, Deutschland: EFI.

Open Access Dieses Kapitel wird unter der Creative Commons Namensnennung 4.0 International Lizenz (http://creativecommons.org/licenses/by/4.0/deed.de) veröffentlicht, welche die Nutzung, Vervielfältigung, Bearbeitung, Verbreitung und Wiedergabe in jeglichem Medium und Format erlaubt, sofern Sie den/die ursprünglichen Autor(en) und die Quelle ordnungsgemäß nennen, einen Link zur Creative Commons Lizenz beifügen und angeben, ob Änderungen vorgenommen wurden.

Die in diesem Kapitel enthaltenen Bilder und sonstiges Drittmaterial unterliegen ebenfalls der genannten Creative Commons Lizenz, sofern sich aus der Abbildungslegende nichts anderes ergibt. Sofern das betreffende Material nicht unter der genannten Creative Commons Lizenz steht und die betreffende Handlung nicht nach gesetzlichen Vorschriften erlaubt ist, ist für die oben aufgeführten Weiterverwendungen des Materials die Einwilligung des jeweiligen Rechteinhabers einzuholen. 